\title{
Control of Nonlinear Instabilities in a System of Coupled Interleaved Buck Converters
}

\author{
I. Daho, D. Giaouris, Member, IEEE B. Zahawi, Senior \\ Member, IEEE, and V. Pickert, Member, IEEE \\ School of Electrical, Electronics and Computer \\ Engineering, Newcastle University, UK. \\ damian.giaouris@ncl.ac.uk
}

\author{
S. Banerjee, Senior Member, IEEE \\ Indian Institute of Technology, Kharagpur-721302 \\ India \\ soumitro@ee.iitkgp.ernet.in
}

\begin{abstract}
Novel controllers to improve the stability of coupled interleaved buck converters operating under current-mode control are proposed in this paper. The controllers are employed to force the eigenvalues of the monodromy matrix of the system (the state transition matrix over one full cycle) inside the unit circle, thus avoiding the Neimark bifurcation which has been known to occur in this system. The indirect coupling between the two inductors employed in the circuit is shown to improve the system's stability. Simulations and Numerical analysis are presented to provide the basic theoretical evidence for the proposed control methods.
\end{abstract}

Keywords-component; Bifurcation control, parallel connected dc-dc converters, monodromy matrix.

\section{INTRODUCTION}

Current mode controlled dc-dc converters are inherently nonlinear and nonsmooth time varying systems. Their nonlinear behavior has been extensively studied in various publications [1-4]. Such works have concentrated only on single-stage topologies. However, when two or more converters are connected in parallel $[5,6]$ their bifurcation behavior is significantly altered. The parallel connection of switching converters is an interesting technique [7] with some advantages over single dc-dc converter designs. Firstly, it allows high load currents to be delivered without employing devices of high power ratings. Secondly, the sharing of the output current between the converters reduces the stress on the switching devices and increases system reliability [8]. The nonlinear behavior of parallel connected dc-dc converters operating with master-slave current-sharing controllers in interleaved current control mode ha been studied in the past $[5,9,10]$. These studies have been based on the conventional Poincare map method which can accurately predict the state of the system for a given set of parameters but offers little knowledge of how and why the loss of stability occurs [11].

In this paper, a different approach is employed, based on Filippov's method, which not only predicts the system loss of stability but also provides a systematic method for developing new control strategies to avoid the onset of the Neimark bifurcation. This method has been previously used by the authors to control the bifurcation behavior of single stage dc$\mathrm{dc}$ converters [11-13]. In this paper we extend the analysis to control the slow scale bifurcation in interleaved, parallel connected dc-dc buck converters. The new method is based on the formulation of the system's monodromy matrix W (the fundamental solution matrix over one full cycle) and using the saltation matrix (the transition matrix at the switching event) to study the flow of the system during the switching instances. The analysis shows how the instability of the circuit can be pinpointed using these matrices and how it can be avoided by appropriately changing their parameters. The effects of introducing indirect coupling between the two inductors on the bifurcation behavior of the system are also discussed.

\section{Parallel ConNected Buck CONVERTERs Using THE INTERLEAVING SWITCHING RULE}

\section{A. Principles of Operation}

The system consists of two identical parallel connected buck converters feeding the same load as shown in Fig. 1. The converters operate in continuous conduction mode. Both converters are controlled using an interleaving switching rule based on a simple peak current controller. The inductor currents $i_{1}$ and $i_{2}$ are compared with a reference current, $I_{\text {ref }}$, to generate the control signals that drive switches $S_{1}$ and $S_{2}$. When $S_{1}\left(S_{2}\right)$ is turned on, $i_{1}\left(i_{2}\right)$ increases until it reaches $I_{r e f}$; then $S_{1}\left(S_{2}\right)$ is turned off and $i_{1}\left(i_{2}\right)$ decreases. $S_{1}\left(S_{2}\right)$ remains off until it is turned on by the periodic clock signal. $S_{1}$ and $S_{2}$ are turned on alternately by the periodic clock signal, i.e. each switch is turned on every $2 T$, as shown in Fig. 2. Diode $D_{i}$ is always in a complementary state to switch $S_{j}(j=1,2)$. Hence, only four switch states are possible during a switching cycle. The state equations that represent these states are: 
2008 IEEE Region 10 Colloquium and the Third International Conference on Industrial and Information Systems, Kharagpur, INDIA December 8 -10, 2008.

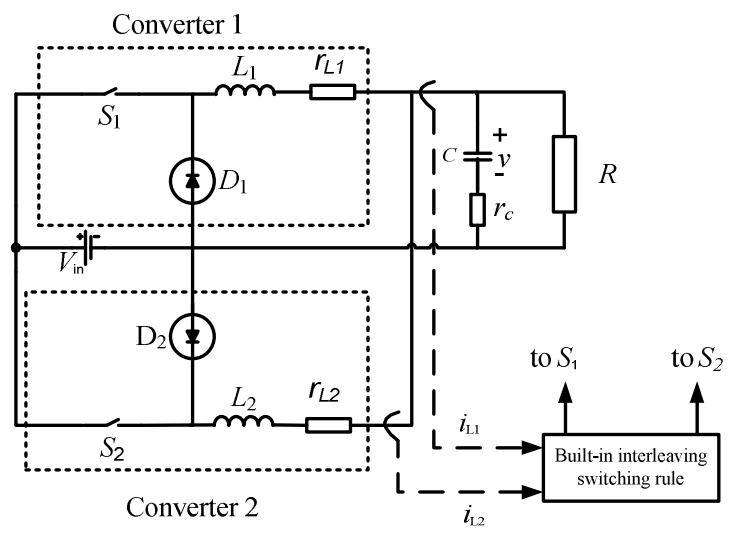

Fig. 1. Parallel-connected buck converters.

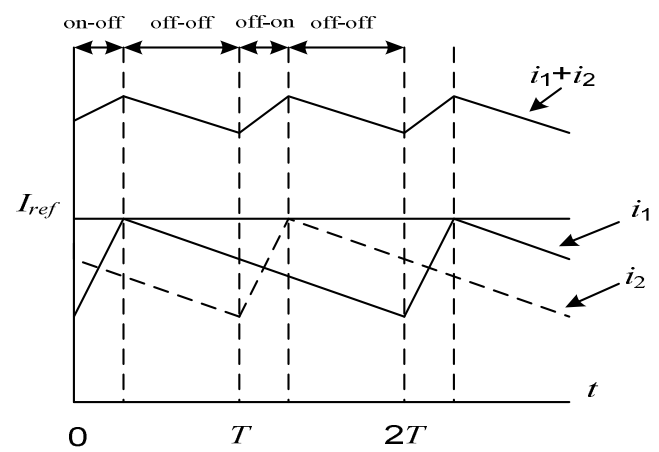

Fig. 2. Current waveforms showing the switching sequence in each switching period.

$$
\begin{array}{ll}
\dot{\mathbf{X}}=\mathbf{A}_{1} \mathbf{X}+\mathbf{B}_{1} V_{\text {in }} & \mathrm{S}_{1} \text { is on and } \mathrm{S}_{2} \text { is on } \\
\dot{\mathbf{X}}=\mathbf{A}_{2} \mathbf{X}+\mathbf{B}_{2} V_{\text {in }} & \mathrm{S}_{1} \text { is on and } \mathrm{S}_{2} \text { is off } \\
\dot{\mathbf{X}}=\mathbf{A}_{3} \mathbf{X}+\mathbf{B}_{3} V_{\text {in }} & \mathrm{S}_{1} \text { is off and } \mathrm{S}_{2} \text { is on } \\
\dot{\mathbf{X}}=\mathbf{A}_{4} \mathbf{X}+\mathbf{B}_{4} V_{\text {in }} & \mathrm{S}_{1} \text { is off and } \mathrm{S}_{2} \text { is off }
\end{array}
$$

where $V_{\text {in }}$ is the input voltage, $\mathbf{X}$ is the state vector defined as $\mathrm{X}=\left[\begin{array}{lll}\mathbf{v} & i_{1} & i_{2}\end{array}\right]^{\mathrm{T}}=\left[\begin{array}{lll}x_{1} & x_{2} & x_{3}\end{array}\right]^{\mathrm{T}}$ and $\mathbf{A}, \mathbf{B}$ are the system matrices. In the case of the buck converters, the system matrices $\mathbf{A}_{1}, \mathbf{A}_{2}$, $\mathbf{A}_{3}$, and $\mathbf{A}_{4}$ are equal to $\mathbf{A}_{\mathrm{S}}$, given by:

$$
\mathbf{A}_{s}=\left[\begin{array}{ccc}
\frac{-1}{C(R+r c)} & \frac{1}{C(R+r c)} & \frac{1}{C(R+r c)} \\
\frac{-R}{L_{1}(R+r c)} & \frac{-1}{L}\left(\frac{r c R}{R+r c}+r_{L 1}\right) & \frac{-1}{L}\left(\frac{r c R}{R+r c}\right) \\
\frac{-R}{L_{2}(R+r c)} & \frac{-1}{L_{2}}\left(\frac{r c R}{R+r c}\right) & \frac{-1}{L_{2}}\left(\frac{r c R}{R+r c}+r_{L 2}\right)
\end{array}\right]
$$

$$
\mathbf{B}_{1}=\left[\begin{array}{c}
0 \\
1 / L_{1} \\
1 / L_{2}
\end{array}\right], \mathbf{B}_{2}=\left[\begin{array}{c}
0 \\
1 / L_{1} \\
0
\end{array}\right], \mathbf{B}_{3}=\left[\begin{array}{c}
0 \\
0 \\
1 / L_{2}
\end{array}\right], \mathbf{B}_{4}=\left[\begin{array}{l}
0 \\
0 \\
0
\end{array}\right]
$$

The control equation can be expressed as:

$$
i_{j}(d 2 T)-I_{r e f}=0, \quad j=1,2
$$

where $d$ is the duty ratio of the converters. Under normal operation, $i_{1}$ and $i_{2}$ are periodic functions with period $2 T$; whereas the output current (the sum of $i_{1}$ and $i_{2}$ ) and the output voltage $v$ are periodic functions with period $T$.

\section{B. Bifurcation Behaviour}

With circuit parameters fixed at $V_{i n}=40 \mathrm{~V}, T=40 \mu \mathrm{s}$, $L_{1}=L_{2}=L=3 \mathrm{mH}, \quad r_{\mathrm{L} 1}=r_{\mathrm{L} 2}=0.05 \Omega, \quad C=4.7 \mu \mathrm{F}, \quad r_{\mathrm{C}}=0.01 \Omega \quad$ and $R=10 \Omega$, exact cycle-by-cycle simulation can be performed using the above equations. A typical bifurcation diagram is shown in Fig. 3, using $I_{\text {ref }}$ as the bifurcation parameter. When $I_{\text {ref }}$ is below $1.16 \mathrm{~A}$ both the output voltage $v$ and the output current $i_{1}+i_{2}$ are stable periodic functions with period $T$, as shown in Fig. 4. Beyond this value of $I_{\text {ref }}$ the system settles into a quasi-periodic (Neimark-Sacker bifurcation) or a higher periodic orbit, as shown in Fig. 5.

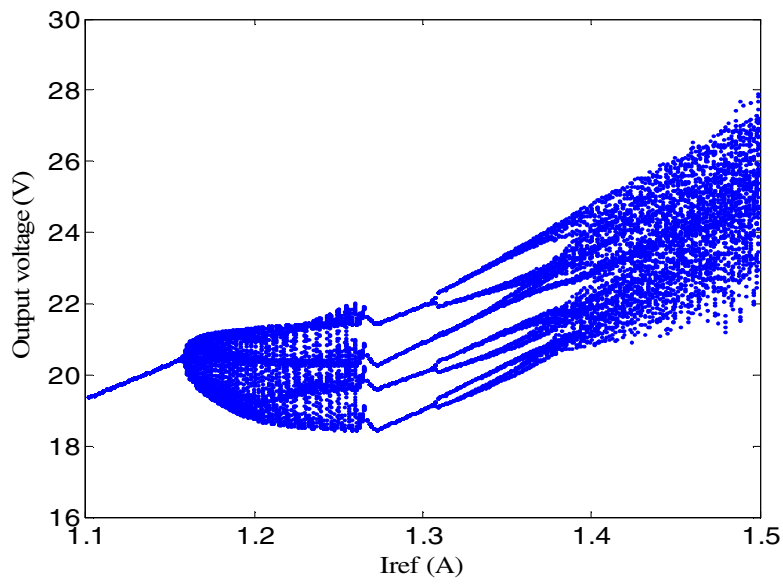

Fig. 3. Bifurcation diagram.

And 
2008 IEEE Region 10 Colloquium and the Third International Conference on Industrial and Information Systems, Kharagpur, INDIA December 8 -10, 2008.

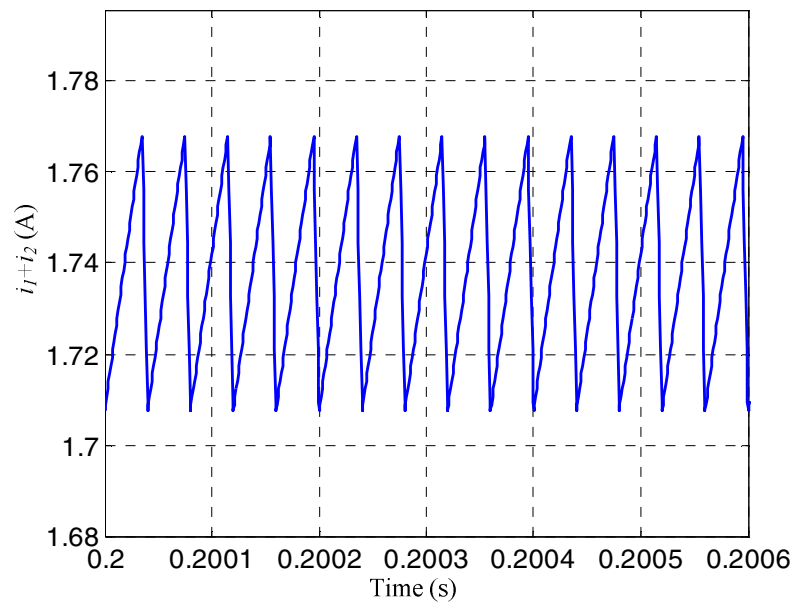

Fig. 4. Current waveform, $I_{\text {ref }}=1.1$ A.

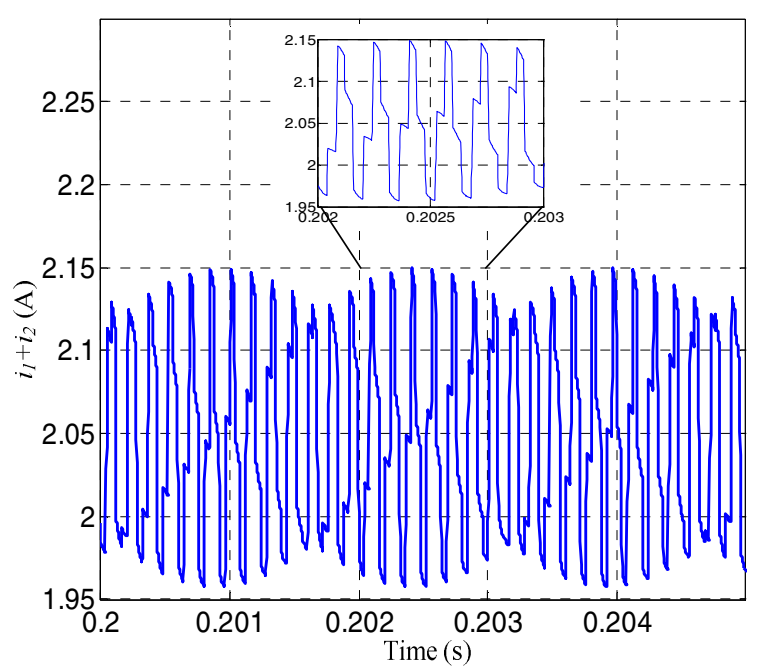

Fig. 5. Current waveform, $I_{\mathrm{ref}}=1.16 \mathrm{~A}$.

\section{STABILITY ANALYSIS}

In this section, the stability of the circuit is analyzed by deriving the eigenvalues of the monodromy matrix (Floqute or characteristic multipliers) of the system. Fig. 6, shows that the trajectory $\left(i_{1}, i_{2}\right)$ crosses the switching manifold four times at $d 2 T, T,(1+2 d) T$ and $2 T$. The monodromy matrix $\mathbf{W}$ of the system for $2 T$ can be expressed as:

$$
\begin{aligned}
\mathbf{W}(0, \mathbf{X}(0), 2 T)= & e^{\mathbf{A}_{s}(d 2 T)} \times \mathbf{S}_{1} \times e^{\mathbf{A}_{s}(0.5-d) 2 T} \\
& \times \mathbf{S}_{2} \times e^{\mathbf{A}_{s}(d 2 T)} \times \mathbf{S}_{3} \times e^{\mathbf{A}_{s}(0.5-d) 2 T} \times \mathbf{S}_{4}
\end{aligned}
$$

where $\boldsymbol{S}_{1}$ and $\boldsymbol{S}_{3}$ are the saltation matrices at $d 2 T$ and $T(1+2 d)$, respectively. $\boldsymbol{S}_{2}$ and $\boldsymbol{S}_{4}$ are the saltation matrices at the switching instant $T$ and $2 T$, respectively, given by the identity matrix [11]. $\boldsymbol{S}_{1}$ and $\boldsymbol{S}_{3}$ can be obtained using the following formula [11]:

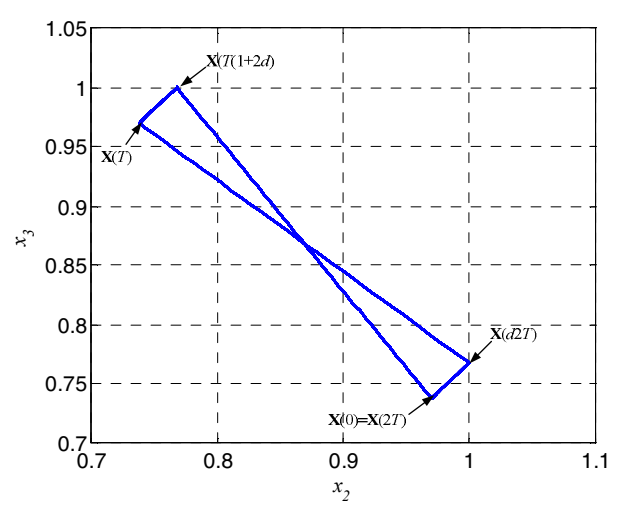

Fig. 6. System trajectory

$$
\mathbf{S}=\mathbf{I}+\left.\frac{\left(\mathbf{f}_{+}(\mathbf{x}(t))-\mathbf{f}_{-}(\mathbf{x}(t))\right) \mathbf{n}^{T}}{\mathbf{n}^{T} \mathbf{f}_{-}(\mathbf{x}(t))+\frac{d h}{d t}}\right|_{t=t_{\Sigma}}
$$

where $h$ is the switching condition, $\mathbf{n}$ is the normal vector to $h$, $t_{\Sigma}$ is the switching time, and $\mathbf{f}$. and $\mathbf{f}_{+}$are the two vector fields before and after the switching manifold. Since the switching equation (1) is not a function of time, the derivative of $h$ with respect to time is zero $(d h / d t=0)$.

At $t_{\Sigma}=d 2 T$ the switching condition is:

$$
h(\boldsymbol{X}(d 2 T))=x_{2}-I_{\text {ref }}
$$

so that $\mathbf{n}_{1}=\left[\begin{array}{lll}0 & 1 & 0\end{array}\right]^{\mathrm{T}}$, and the two vector fields before and after the switching are:

$$
\begin{aligned}
& \mathbf{f}_{-}(\mathbf{X}(d 2 T))=\mathbf{A}_{s} \mathbf{X}(d 2 T)+\mathbf{B}_{2} V_{i n} \\
& \mathbf{f}_{+}(\mathbf{X}(d 2 T))=\mathbf{A}_{s} \mathbf{X}(d 2 T)+\mathbf{B}_{4} V_{i n}
\end{aligned}
$$

Similarly, at $t_{\Sigma}=\mathrm{T}(1+2 d)$ the switching condition is:

$$
h\left(X(T(1+2 d))=x_{3}-I_{r e f}\right.
$$

so that $\mathbf{n}_{2}=\left[\begin{array}{lll}0 & 0 & 1\end{array}\right]^{\mathrm{T}}$, and the two vector fields before and after the switching are:

$$
\begin{aligned}
& \mathbf{f}_{-}(\mathbf{X}(T(1+2 d)))=\mathbf{A}_{s} \mathbf{X}(T(1+2 d))+\mathbf{B}_{3} V_{i n} \\
& \mathbf{f}_{+}(\mathbf{X}(T(1+2 d)))=\mathbf{A}_{s} \mathbf{X}(T(1+2 d))+\mathbf{B}_{4} V_{i n}
\end{aligned}
$$


2008 IEEE Region 10 Colloquium and the Third International Conference on Industrial and Information Systems, Kharagpur, INDIA December 8 -10, 2008.

TABLE I

EIGENVALUES FOR VARIOUS $\mathrm{I}_{\text {REF }}$

\begin{tabular}{|c|c|c|c|c|}
\hline \hline$I_{\text {ref }}$ & \multicolumn{2}{|c|}{ Eigenvalues } & $\begin{array}{c}\text { Modulus of } \\
\text { Complex pair }\end{array}$ & \\
\hline 1.1 & 0.1992 & $-0.8690 \pm 0.0813 \mathrm{i}$ & 0.8690 & Period-1 \\
\hline 1.12 & 0.2005 & $-0.9292 \pm 0.0826 \mathrm{i}$ & 0.9328 & Period-1 \\
\hline 1.14 & 0.2016 & $-0.9637 \pm 0.0837 \mathrm{i}$ & 0.9666 & Period-1 \\
\hline 1.16 & 0.2028 & $-0.9998 \pm 0.0848 \mathrm{i}$ & 1.0034 & Neim.Bif \\
\hline 1.18 & 0.204 & $-1.0374 \pm 0.0859 \mathrm{i}$ & 1.041 & Unstable \\
\hline 1.2 & 0.2051 & $-1.0767 \pm 0.0868 \mathrm{i}$ & 1.0802 & Unstable \\
\hline \hline
\end{tabular}

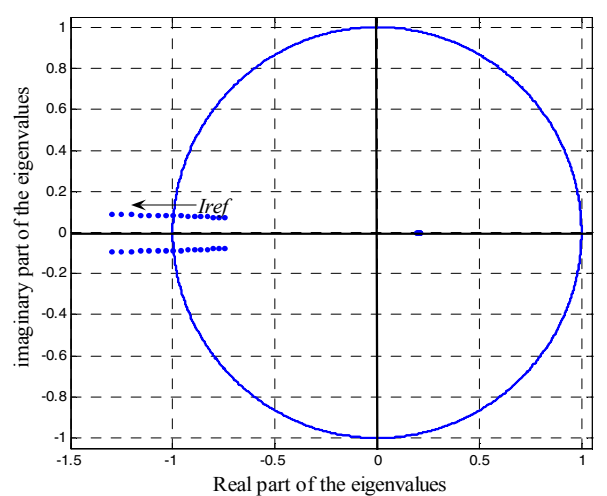

Fig. 7. The loci of the eigenvalues of the monodromy matrix.

The stability of the system can be determined by finding the eigenvalues of the monodromy matrix $\mathbf{W}$ :

$$
\operatorname{det}[\lambda \mathbf{1}-\mathbf{W}]=0
$$

To calculate $\mathbf{W}$, and hence evaluate the stability of the system, we need to numerically calculate the values of the state vectors $\mathbf{X}(0), \mathbf{X}(d 2 T)$ and $\mathbf{X}(T(1+2 d))$ [5]. Table 1 shows the eigenvalues of the monodromy matrix as $I_{r e f}$ is varied. The computed loci of the eigenvalues with varying $I_{r e f}$ are shown in Fig. 7. The system lose stability through a Neimarkbifurcation (the complex pair of eigenvalues moves out of the unit circle) at a value of $I_{\text {ref }}$ of around 1.16 A. This result is in very good agreement with the simulation results presented in Fig. 5, as well as the results produced using the conventional Poincare map method [5].

\section{CONTROLling The BifurCATION}

It can be observed from the previous section that the system starts to leave the stable period 1 region through a Neimark bifurcation. In this section we will show how this region can be extended by controlling the Neimark bifurcation based on our knowledge of the saltation matrix. Equation (2) can be written as:

$$
\mathbf{W}(0, \mathbf{X}(0), 2 T)=e^{\mathbf{A}_{s} T} \times \mathbf{S}_{1} \times e^{\mathbf{A}_{s} T} \times \mathbf{S}_{3}
$$

From (11) it is clear that the monodromy matrix is a function of the saltation matrices, as $e^{\mathbf{A} s T}$ is constant. Therefore, the saltation matrices $\mathbf{S}_{1}$ and $\mathbf{S}_{3}$ play a central role in determining the eigenvalues of the monodromy matrix and hence the stability of the system. This implies that the saltation matrix is mainly responsible for the occurrence of the bifurcation in coupled interleaved buck converters. It is therefore of interest to study the structure of this matrix (3) and see if we can reverse this change to stabilize the unstable period 1 orbit. The saltation matrix is clearly a function of the two vector field before and after the switching off instant and of the manifold $h$. Hence, we can alter the saltation matrix either by appropriately introducing small changes in the derivative of $h$ with respect to time (making $d h / d t$ nonzero), or in the normal vector $\mathbf{n}$. In the following subsections, two alternative methods are proposed to guarantee stability over a wider range of $I_{\text {ref }}$ values.

\section{A. Control Based on Changing the Derivative of $h$ With Respect to Time}

To demonstrate this idea, we add a sinusoidal signal with amplitude $a$ to $I_{\text {ref }}[14]$, such that:

$$
\begin{gathered}
h_{1}(\mathbf{X}, t)=x_{2}-\left.I_{r e f}(1+a \sin (t))\right|_{t=d 2 T} \\
h_{2}(\mathbf{X}, t)=x_{2}-\left.I_{r e f}(1+a \sin (\omega t))\right|_{t=T(1+2 d)}
\end{gathered}
$$

Therefore:

$\left.\frac{d h}{d t}=\omega I_{r e f} a \cos (\omega d 2 T)\right)$ where $\omega=2 \pi / T$.

$\mathbf{S}_{1}$ and $\mathbf{S}_{2}$ are now given by:

$$
\mathbf{S}_{1}=\left[\begin{array}{ccc}
1 & 0 & 0 \\
0 & 1-\frac{V_{\text {in }}}{L} Y_{1} & 0 \\
0 & 0 & 1
\end{array}\right] \mathbf{S}_{3}=\left[\begin{array}{ccc}
1 & 0 & 0 \\
0 & 1 & 0 \\
0 & 0 & 1-\frac{V_{\text {in }}}{L} Y_{2}
\end{array}\right]
$$

where

$$
Y_{1}=\left(\mathbf{n}_{1}{ }^{T} \mathbf{f}_{-}\left(\mathbf{x}(d 2 T)+\omega I_{r e f} a \cos (\omega d T)\right)\right)^{-1}
$$

$$
Y_{2}=\left(\mathbf{n}_{2}{ }^{T} \mathbf{f}_{-}\left(\mathbf{x}(T(1+2 d))+\omega I_{r e f} a \cos (\omega d 2 T)\right)\right)^{-1}
$$

The effect of this change can be better understood by studying the terms of the saltation matrix. Notice that $\sin (\omega t)$ at $d 2 T$ and at $T(1+2 d)$ is very small; hence its influence on $h_{l}$ and $h_{2}$ will be very small. However, its derivative with respect 
2008 IEEE Region 10 Colloquium and the Third International Conference on Industrial and Information Systems, Kharagpur, INDIA December 8 -10, 2008.

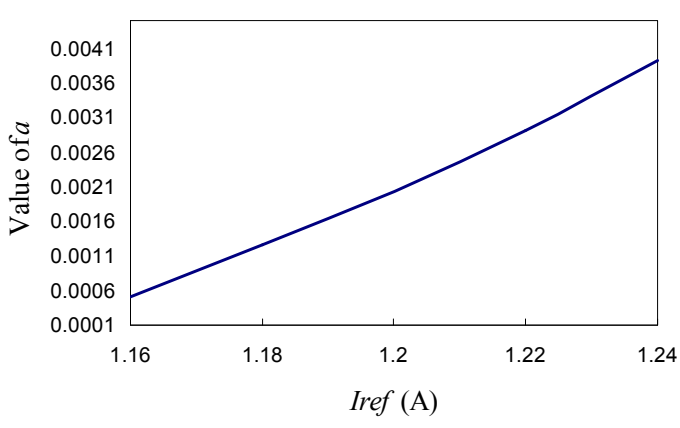

Fig. 8: Values of $a$ needed to maintain stability.

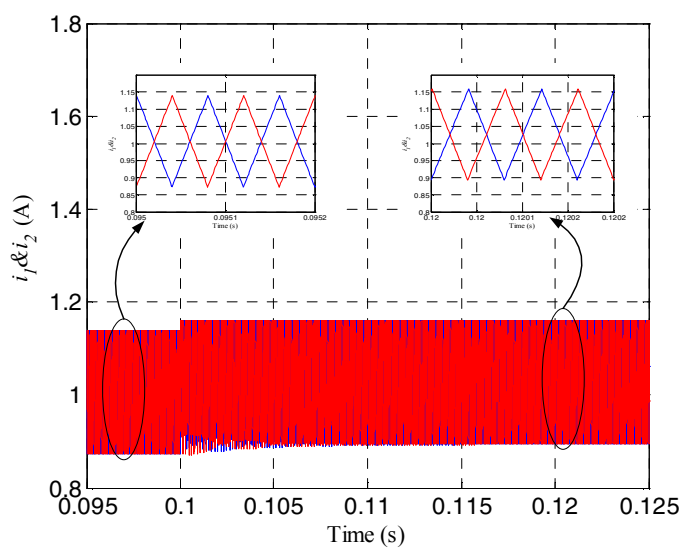

Fig. 9. Controller response; sinusoidal signal injection.

to time will be relatively large and will have a significant effect on the saltation matrices $\mathbf{S}_{1}$ and $\mathbf{S}_{2}$. It is obvious from (14) and (15) that the eigenvalues will be a function of $a$. Thus, by altering the value of $a$ it is possible to affect the eigenvalues of the monodromy matrix and hence the stability of the system. To design a suitable controller we can numerically solve the nonlinear transcendental equation (16) to maintain the absolute value of the complex eigenvalues at say 0.9780 (Fig. 8).

$$
|0.9780 \times I-\mathbf{W}|=0
$$

The response of this controller as $I_{\text {ref }}$ is increased is shown in Fig. 9. It is clear that after an initial transient the system will settle down to the stable period 1 limit cycle and stability is retained.

\section{B. Control Based on Changing the Derivative of $h$ With Respect to the State Vector $d h / d\left(x_{1}\right)$}

This can be achieved by adding a small voltage feedback signal to the current reference, such that:

$$
h_{1}(\mathbf{X}, t)=x_{2}(d 2 T)-\left.\left(I_{r e f}-b x_{1}(d 2 T)\right)\right|_{t=d 2 T}
$$

$$
\frac{d h_{1}}{d x_{1}}=\mathbf{n}_{1}=\left[\begin{array}{lll}
b & 1 & 0
\end{array}\right]^{T}
$$

Hence:

$$
\mathbf{S}_{1}=\left[\begin{array}{ccc}
1 & 0 & 0 \\
-b \frac{V_{\text {in }}}{L} & 1-\frac{V_{\text {in }}}{L} Y_{1} & 0 \\
0 & 0 & 1
\end{array}\right] \mathbf{S}_{3}=\left[\begin{array}{ccc}
1 & 0 & 0 \\
0 & 1 & 0 \\
-b \frac{V_{\text {in }}}{L} & 0 & 1-Y_{2} \frac{V_{\text {in }}}{L}
\end{array}\right]
$$

where

$$
\begin{gathered}
Y_{1}=\left(\mathbf{n}_{1}{ }^{T} \mathbf{f}_{-}(\mathbf{x}(d 2 T))\right)^{-1} \\
Y_{2}=\left(\mathbf{n}_{2} \mathbf{f}_{-}(\mathbf{x}(T(1+2 d)))^{-1}\right.
\end{gathered}
$$

It is obvious that the two saltation matrices are a function of $b$. The values of $b$ needed to maintain stability can also be calculated by numerically solving the nonlinear transcendental equation (16) to once more maintain the absolute value of the complex eigenvalues at 0.9780 (Fig. 10). The response of this controller is shown in Fig. 11.

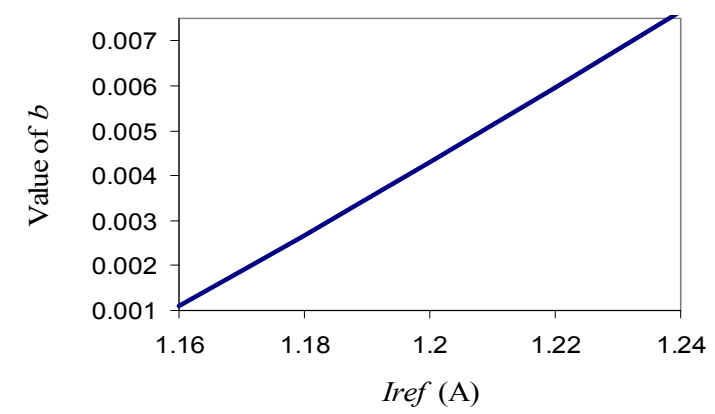

Fig. 10. Values of $b$ needed to maintain stability.

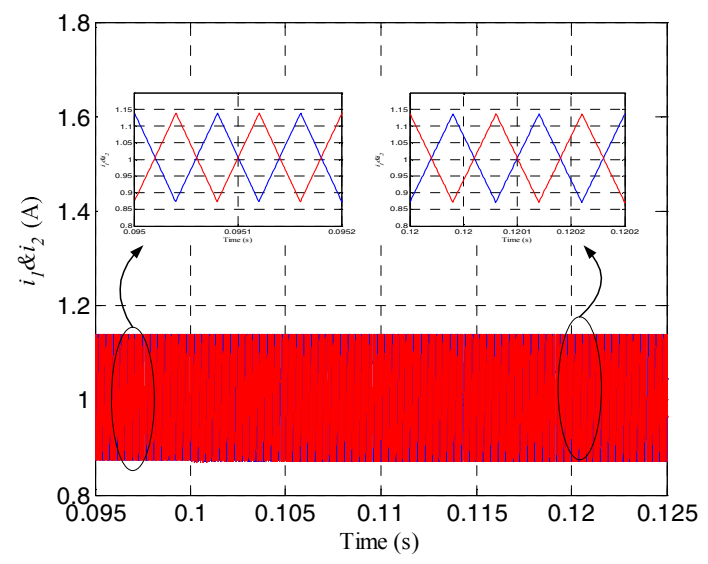

Fig. 11. Controller response; voltage feedback signal.

where $b$ is a small control parameter. 
2008 IEEE Region 10 Colloquium and the Third International Conference on Industrial and Information Systems, Kharagpur, INDIA December 8 -10, 2008.

367

TABLE 2

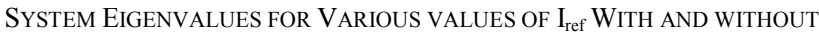
MutUAL COUPLING EFFECTS BETWEEN THE TWO INDUCTORS

\begin{tabular}{|c|c|c|c|}
\hline \hline$I_{\text {ref }}$ & $\alpha$ & $\begin{array}{c}\text { Modulus with mutual } \\
\text { inductance effects }\end{array}$ & $\begin{array}{c}\text { Modulus } \\
\text { without mutual } \\
\text { inductance effects }\end{array}$ \\
\hline 1.16 & -0.079 & 0.9782 & 1.0034 \\
\hline 1.18 & -0.174 & 0.978 & 1.041 \\
\hline 1.12 & -0.25 & 0.9781 & 1.0802 \\
\hline \hline
\end{tabular}

\section{I HE EFFEC"I OF LOUPLING I HE I WO INDUC"IORS}

Interleaved switching and coupled inductors are an attractive modification as they improve the output waveform quality in the parallel connected dc-dc converter [15]. To study the effects of coupling between the two inductors, the two saltation matrices $\mathbf{S}_{1}$ and $\mathbf{S}_{3}$ were modified to account for mutual inductance effects.

$$
\mathbf{S}_{1}=\left[\begin{array}{ccc}
1 & 0 & 0 \\
0 & 1-\frac{V_{\text {in }}}{L \pm M} Y_{1} & 0 \\
0 & 0 & 1
\end{array}\right] \mathbf{S}_{3}=\left[\begin{array}{ccc}
1 & 0 & 0 \\
0 & 1 & 0 \\
0 & 0 & 1-\frac{V_{\text {in }}}{L \pm M} Y_{2}
\end{array}\right]
$$

The saltation matrices are now a function of the mutual inductance $M$. This can either improve the system stability or make it worse depending on the polarity of the magnetic coupling between the two inductors. Table 2 shows that the system stability can be improved by introducing indirect coupling (negative $M$ ) into the system.

\section{CONCLUSION}

A new method for controlling the slow scale bifurcation in the interleaved parallel connected buck converters operating under current mode control have been proposed in this paper. Two controllers have been proposed based on the simple idea of altering the parameters of the saltation matrices to influence bifurcation control law. The derived saltation matrices were also modified to allow for the effects of mutual coupling between the two converter inductors. It has been shown that introducing indirect coupling between the inductors can increase the stability of the system and extend the stable period 1 region of operation.

\section{REFERENCES}

[1] G. C. Verghese and S. Banerjee, Nonlinear Phenomena in Power Electronics: Bifurcations, Chaos, Control, and Application. New York: IEEE Press, 2001.

[2] K. Chakrabarty, G. Poddar and S. Banerjee, "Bifurcation behavior of the buck converter," Power Electronics, IEEE Transactions on, vol. 11, pp. 439-447, 1996.

[3] S. Banerjee and K. Chakrabarty, "Nonlinear modeling and bifurcations in the boost converter," Power Electronics, IEEE Transactions on, vol. 13, pp. 252-260, 1998.

[4] C. K. Tse and M. Di Bernardo, "Complex behavior in switching power converters," Proceedings of the IEEE, vol. 90, pp. 768-781, 2002.

[5] H. H. C. Iu, C. K. Tse and O. Dranga, "Comparative study of bifurcation in single and parallel-connected buck converters under current-mode control: disappearance of period-doubling," Circuits Systems Signal processing vol. 24, pp. 201-219, 2005/04 2005.

[6] Y. Ishikawa and T. Saito, "Synchronization and Chaos in MultipleInput Parallel DC-DC Converters with WTA Switching," IEICE Transactions on Fundamentals of Electronics, Communications and Computer Sciences, vol. E90-A, pp. 1162-1169, 2007.

[7] T. Saito, S. Tasaki and H. Torikai, "Interleaved Buck Converters Based on Winner-Take-All Switching," Circuits and Systems I: Regular Papers, IEEE Transactions on [Circuits and Systems I: Fundamental Theory and Applications, IEEE Transactions on], vol. 52, pp. 1666$1672,2005$.

[8] A. N. Natsheh and J. M. Nazzal, "Application of bifurcation theory to current-mode controlled parallel-connected DC-DC boost converters with multi bifurcation parameters," Chaos, Solitons \& Fractals, vol. 33, pp. 1135-1156, 2007.

[9] H. H. C. Iu and C. K. Tse, "Bifurcation behavior in parallel-connected buck converters," Circuits and Systems I: Fundamental Theory and Applications, IEEE Transactions on [see also Circuits and Systems I: Regular Papers, IEEE Transactions on], vol. 48, pp. 233-240, 2001.

[10] H. H. C. Iu, C. K. Tse , V. Pjevalica and Y. M. Lai, "Bifurcation behaviour in parallel-connected boost converters," International Journal of Circuit Theory and Applications, vol. 29, pp. 281-298, 2001.

[11] D. Giaouris, S. Banerjee, B. Zahawi and V. Pickert, "Stability Analysis of the Continuous Conduction Mode Buck Converter via Filippov's Method," IEEE Transactions on circuits and systems-, vol. 55, pp. 1084-1096, 2008.

[12] D. Giaouris, S. Banerjee, B. Zahawi and V. Pickert, "Control of Fast Scale Bifurcations in Power-Factor Correction Converters," IEEE transaction on circuit and system-II, vol. 54, pp. 805-809, 2007.

[13] I. Daho, D. Giaouris, B.Zahawi, P. Volker and S. Banerjee, "Stability Analysis and Bifurcation Control of Hysteresis Current Controlled Cuk Converter Using Filippov's Method," PEMD 2008, IET International Conference on Power Electronics Machines and Drives, pp. 381-385, York, UK, 2008.

[14] Y. Zhou, C. K. Tse, S. S. Qiu and F. C. M. Lau, "Applying Resonant Parametric Perturbation to Control Chaos in the Buck Dc/Dc Converter With Phase Shift and Frequency Mismatch Considerations," Int. J. Bifurc. Chaos, vol. 13, pp. 3459-3471, 2003.

[15] W. Pit-Leong, X. Peng, P. Yang and F. C. Lee, "Performance improvements of interleaving VRMs with coupling inductors," IEEE Transactions on Power Electronics, vol. 16, pp. 499-507, 2001. 